\title{
Study on Analysis of Peripheral Biomarkers for Alzheimer's Disease Diagnosis
}

\author{
Palaniswamy Rani*, Sreeram Krishnan and Chellappa Rani Cathrine \\ Department of Biotechnology, PSG College of Technology, Coimbatore, India
}

Many factors are involved in Alzheimer's disease (AD) pathology including tau phosphorylation, amyloid $\beta$ protein $(A \beta)$ accumulation, lipid dysregulation, oxidative stress, and inflammation. The markers of these pathological processes in cerebral spinal fluid are used currently for AD diagnosis. However, peripheral biomarkers are the need of the hour for large population screening for AD. The main objective of the present study is to evaluate the peripheral levels of redox markers, lipid peroxidation (LPO) indicators, and pathological markers in AD patients. Blood was collected from AD patients $(n=45)$, controls $(n=45)$, and analyzed for pathological markers of $A D$ including $A \beta 42$ and

OPEN ACCESS

Edited by:

Saradhadevi Varadharaj, Abbott, United States

Reviewed by:

Rami N. Khayat,

The Ohio State University

Columbus, United States

Suowen Xu,

University of Rochester,

United States

Noah Lucas Weisleder,

The Ohio State University Columbus, United States

*Correspondence:

Palaniswamy Rani rani@bio.psgtech.ac.in

Specialty section:

This article was submitted to Neurodegeneration, a section of the journal Frontiers in Neurology

Received: 28 February 2017 Accepted: 22 June 2017 Published: 14 July 2017

Citation:

Rani P. Krishnan S and Rani Cathrine C (2017) Study on Analysis of Peripheral Biomarkers for Alzheimer's Disease Diagnosis.

Front. Neurol. 8:328. doi: 10.3389/fneur.2017.00328 tau, LPO, and redox indicators. Plasma A $\beta 42$ was significantly $(P<0.001)$ elevated while total tau was decreased in AD compared to controls. Hydroxynonenal (HNE) and malondialdehyde (MDA) were higher $(P<0.001)$ in AD patients pointing the enhanced $L P O$ in AD pathology. Receiver operating characteristic curve $(R O C)$ analysis indicated that HNE is a better indicator of LPO compared to MDA. Plasma glutathione (GSH) level was significantly $(P<0.001)$ low while oxidized glutathione (GSSG) level was higher $(P<0.001)$ in AD patients with corresponding decrease in GSH/GSSG ratio $(P<0.001)$. $\mathrm{ROC}$ analysis indicated that GSH/GSSG ratio can be used as reliable indicator for redox imbalance in $A D$ with a cutoff value of $<8.73$ (sensitivity $91.1 \%$, specificity $97.8 \%$ ). Correlation analysis revealed a positive correlation for both HNE and MDA with A $\beta 42$ and a negative correlation with total tau. Negative correlation was observed between GSH/ GSSG ratio and LPO markers. While oxidative stress has been implicated in pathology of various neurodegenerative disorders, the present study pinpoints the direct link between LPO and A $\beta$ production in plasma of AD patients. Normally, at low amyloid concentration in body fluids, this peptide shown to function as a strong metal chelating antioxidant. However, when the $A \beta$ production enhanced as in $A D$, through gain of functional transformation, $A \beta$ evolves into prooxidant, thereby enhancing oxidative stress and LPO. Altered redox status with enhanced LPO observed in AD blood could contribute to the oxidation and $S$-glutathionylation proteins, which has to be addressed in future studies.

Keywords: Alzheimer's disease, oxidative stress, biomarkers, redox status, lipid peroxidation

\section{INTRODUCTION}

Alzheimer's disease (AD) is a debilitating illness of the nervous system, affecting millions of elderly individuals worldwide. Numerous factors are involved in the disease etiology including tau phosphorylation, amyloid $\beta$ protein (A $\beta$ ) accumulation, lipid dysregulation, oxidative stress, and inflammation. Among all factors, oxidative stress plays a central role in the pathogenesis of $\mathrm{AD}$ 
leading to neuronal dysfunction and cell death (1). Oxidative stress is caused by increased production of reactive oxygen species (ROS), which denature biomolecules such as proteins, lipids, and nucleic acids through pathological redox reactions (2). Increased oxidative stress also results in excessive lipid peroxidation (LPO), which in turn perturb the bilayer structure and modify the membrane properties such as membrane fluidity, permeability, and bilayer thickness (3). This might cause an imbalance in the concentration of ions and impairing metabolism, thereby leading to apoptosis and necrosis. Oxidative stress has been emphasized in pathology of various inflammatory diseases including cancer, atherosclerosis, diabetes, and neurodegenerative disorders (4). However, human brain is more vulnerable to oxidative stress due to high oxygen consumption and high concentration of easily oxidizable polyunsaturated fatty acid. Particularly, LPO markers are found to be elevated in brain tissue and fluid in several neurodegenerative disorders. The status of LPO has extensively analyzed in AD, PD. There are also controversy regarding the LPO marker in neurodegenerative disorder, whether it represents the distinct pathomechanism of the specific disease or merely a non-specific occurrence (5).

The human body is enriched with many endogenous antioxidants to scavenge excess ROS produced. Antioxidant enzymes like peroxidase, superoxide dismutase along with glutathione (GSH), help in nullifying the ill effects of excess ROS. While the levels of these antioxidants are shown to be elevated in $\mathrm{AD}$ brain owing to neuronal damage, increase in the levels of peripheral antioxidants observed indicate that the damage is not limited to brain alone $(6,7)$. Alteration in levels of circulating antioxidants is also directly related to the severity of cognitive impairment (8). Elevated levels of markers of LPO including hydroxynonenal (HNE) and malondialdehyde (MDA) were reported in AD $(9,10)$.

Reduced glutathione (GSH), in parallel with oxidized glutathione (GSSG), is generally responsible for maintaining redox balance of the cell. Mandal et al. (11) reported a reduction in GSH levels in the brain of AD patients along with an alteration in GSH/GSSG ratio, indicating excessive oxidative damage. Moreover, incidence of increased protein glutathionylation has also been reported in the inferior parietal lobule of $\mathrm{AD}$ brain (12). Estimating the markers of LPO and redox status over a period of time would be helpful in assessing the disease progression. Since AD is a brain-related disease, only a little evidence is present on the peripheral manifestation. Research on identifying the blood-related biomarkers of $\mathrm{AD}$ is in fast progress since the determination of biomarkers in cerebral spinal fluid (CSF) is invasive, expensive, and time-consuming. Although amyloid beta 1-42 (A $\beta 42)$ and total tau in blood are being currently considered for diagnosis of $\mathrm{AD}$ (13), there is a need to determine other peripheral markers of AD because of the multiple pathological processes involved. Thus, the present study is designed with a principal objective to assess the peripheral LPO and redox markers in $\mathrm{AD}$, and correlating their levels with the pathological markers in peripheral blood in $\mathrm{AD}$ patients. The study was focused on identification of a panel of oxidative stress markers in blood along with $A \beta 42$ and tau for diagnosis of AD.

\section{MATERIALS AND METHODS}

\section{Study Population}

The present study was approved by the Institutional Ethical Committee of PSG Institute of Medical Sciences and Research (PSG IMS\&R), Madras Medical College and Government General Hospital (MMC), Chennai, and all procedures involving human subject complied with the Declaration of Helsinki. Informed consent was obtained from control subject and from the relatives, legal guardians of the AD patients before their inclusion in the study. The AD patients $(n=45)$ involved in the study were recruited from MMC, Chennai, PSG IMS\&R, Coimbatore, and Alzheimer's and Related Disorders Society of India (ARDSI), Thrissur, India. The cognitive status of the subject was assessed using Mini-Mental State Examination (MMSE) (14), and categorized according to the criteria of NINCDS-ADRDA and DSM-IV for dementia (15). The AD patients with cardiovascular disease and diabetes were excluded in the study. The control subject $(n=45)$ included in the study were in the age group of 50-70 years with a negative history of cardiovascular disorders, diabetes, and neurological disorders. Subject with a habit of smoking, alcohol consumption, or exhibiting comorbidity with advanced malignancies, AIDS, and other major illness were excluded from the study.

\section{Sample Collection}

Blood samples $(10 \mathrm{~mL})$ were collected from each study subject in EDTA tubes and plasma was separated by centrifugation at 2,500 $3,000 \mathrm{rpm}$ for $15 \mathrm{~min}$. The separated plasma samples were aliquoted in microfuge tubes $(0.5 \mathrm{~mL})$ and stored at $-20^{\circ} \mathrm{C}$ for further use.

\section{Pathological Markers of AD}

A $\beta 42$ and total tau levels in plasma were quantified using a commercially available ELISA kit from CUSABIO, China (CSBE10684h, CSB-E12011h). The sandwich ELISA procedure was employed in these kits and the yellow color developed because of horse radish peroxidase reaction was read at $450 \mathrm{~nm}$ with a wavelength correction of $540 \mathrm{~nm}$ in a plate reader.

\section{LPO Markers}

Lipid peroxidation markers including HNE and MDA were evaluated in the present study. The assay of MDA was performed by thiobarbituric acid (TBA) assay method described by Buege and Aust (16). The reaction of MDA with TBA formed a red-colored MDA-TBA complex, which was measured at $535 \mathrm{~nm}$. HNE was determined in plasma samples as described by Benedetti et al. (17). The method involves the carbonyl derivatization of samples with 2,4-dinitrophenylhydrazine, thin-layer chromatographic separation of 2,4-dinitrophenylhydrazine carbonyls, and HPLC-based analysis of dinitrophenylhydrazone of HNE, which was detected at $378 \mathrm{~nm}$.

\section{Redox Markers}

The oxidized and reduced glutathione was measured using the colorimetric method of analysis (18). Reduced glutathione (GSH) reacts with Ellman's Reagent [5,5' -dithio-bis-(2-nitrobenzoic acid)] DTNB to generate 2-nitro 5-thiobenzoic acid, which 
was determined by measuring absorbance at $412 \mathrm{~nm}$. The assay of oxidized glutathione (GSSG) is based on the glutathione recycling system using glutathione reductase (GR) and DTNB (19).

\section{Statistical Analysis}

The results were expressed as a mean \pm SD. Statistical analysis was performed using the statistical package SPSS Version.16, Chicago, IL, USA. All parameters were under the normal distribution, except for the amyloid and tau, for which MannWhitney $U$-test was performed to compare between $\mathrm{AD}$ and control. Receiver operating characteristic (ROC) curve analysis was performed to study the reliability of HNE, MDA, and GSH/ GSSG ratio in plasma as markers for AD diagnosis. Pearson's correlation was done wherever applicable.

\section{RESULTS}

The products of pathophysiological processes in $\mathrm{AD}$ brain are shown to diffuse to the blood (20). This study focuses on analysis of such products in plasma of AD patients, which could lead to the identification of reliable blood-based biomarkers, which would serve to reflect the underlying AD pathology.

The AD patients and controls involved in the study were in the age group of $60-80$ years. The mean age of $\mathrm{AD}$ patients is $71.73 \pm 8.84$ years and the control is $67.46 \pm 8.29$. Assessment of the cognitive status of the subject indicated that $\mathrm{AD}$ patients exhibited profound to severe loss in their cognitive abilities (Table 1).

\section{Pathological Markers of AD}

The CSF tau and A $\beta 42$ are the biochemical markers currently used in AD diagnosis (21). Since diagnosis could mainly rely on plasma markers, the plasma levels of pathological markers of $\mathrm{AD}$, including total tau and A $\beta 42$ were evaluated. The plasma A $\beta 42$ level was found to be twofold higher in AD compared to control. Whereas total tau was found to be decreased by 1.8 -fold in $\mathrm{AD}$ patients. The increased A $\beta 42$ levels with decreased tau resulted in corresponding decrease in tau-amyloid ratio in $\mathrm{AD}$ patients compared to control group and this variation was statistically significant $(P<0.001)$ (Table 2$)$. There was no significant association observed between pathological markers and MMSE score (Amyloid $r=-0.054 ; P>0.05$ and total tau $r=-0.085 ; P>0.05$ ).

\section{Peripheral LPO Markers in AD}

Reactive-free radicals alter phospholipids structure and function in cell membranes leading to the formation of lipid peroxides,

TABLE 1 | Cognitive status of Study group.

\begin{tabular}{lcc}
\hline Subject $(\boldsymbol{n}=\mathbf{4 5})$ & Mean age (years) & Mean MMSE \\
\hline $\begin{array}{l}\text { Alzheimer's disease }(\text { male }=24 ; \\
\text { female }=21)\end{array}$ & $71.73 \pm 8.84$ & $3.50 \pm 2.5$ \\
Controls $($ male $=22$; female $=23)$ & $67.46 \pm 8.29$ & $28.15 \pm 2.60$ \\
\hline
\end{tabular}

Values are expressed as mean $\pm S D$

Mini Mental State Examination (MMSE) score used for measuring the degree of

cognitive impairment with the following scale.

0-4, $\rightarrow$ profound; 4-13, $\rightarrow$ severe; 13-16, $\rightarrow$ moderate; 17-23, $\rightarrow$ mild; 24-30, $\rightarrow$ normal. generating the various small compounds namely MDA and HNE. These two compounds are assessed routinely as indicators of LPO index. In the present study, HNE and MDA were analyzed in plasma of $\mathrm{AD}$ patients to assess the oxidative damage in the cellular system. Significant increase in $\operatorname{HNE}(0.38 \pm 0.26 \mu \mathrm{M})$ level was observed in plasma of AD patients compare to control group $(0.08 \pm 0.05 \mu \mathrm{M} ; P<0.001)$. The MDA level $(2.38 \pm 0.44 \mu \mathrm{M})$ was also found increased in plasma samples of $\mathrm{AD}$ patients $(1.78 \pm 0.33 \mu \mathrm{M} ; P<0.001)$. The results indicated that both HNE and MDA were elevated in $\mathrm{AD}$ patients pointing the enhanced LPO in AD (Figure 1). ROC analysis was performed to validate the use of both HNE and MDA as plasma markers of AD. It was observed that HNE was a better indicator of LPO in AD with a cut-off value of $>0.105 \mu \mathrm{M}$ (sensitivity $93.5 \%$, specificity $75 \%$ ) compared to MDA (cutoff: $>1.98 \mu \mathrm{M}$, sensitivity $80 \%$, specificity $75 \%$ ) (Figure 2). Among the plasma pathological markers of AD, A $\beta 42$ peptide showed positive correlation with $\mathrm{HNE}(r=0.423$; $P<0.01)$ and MDA $(r=0.423 ; P<0.01)$ whereas negative correlation was observed for total tau with $\operatorname{HNE}(r=-0.370$; $P<0.01)$ and MDA $(r=-0.291 ; P<0.01)$ (Figure 3). However, no significant correlation was observed between MMSE and LPO markers including HNE $(r=0.184 ; P>0.05) \mathrm{MDA}(r=0.128$; $P>0.05)$.

\section{Plasma Redox Markers in AD}

To evaluate the redox status of the peripheral system, GSH, GSSG, and GSH/GSSG ratio were analyzed in the plasma of $\mathrm{AD}$ patients. The results revealed that GSH was significantly reduced by $84 \%(P<0.001)$ while GSSG was increased by $74 \%$ $(P<0.001)$ in $\mathrm{AD}$ patients compared to controls contributing to the corresponding decrease of about $54 \%$ in GSH/GSSG ratio $(P<0.001)$ correspondingly (Figure 4$)$. The changes observed in the redox status of the blood were correlated with increased LPO markers. Negative correlation was observed between GSH/ GSSG ratio and LPO markers HNE $(r=-0.356 ; P<0.01)$ MDA $(r=-0.368 ; P<0.01)$ (Figure 5). GSH/GSSG ratio also correlated with AD pathological markers namely A $\beta 42(r=-0.291$; $P<0.01)$ and total tau $(r=0.483 ; P<0.01)$ (Figure 6). ROC analysis indicated that GSH/GSSG ratio can be used as reliable marker for the diagnosis of $\mathrm{AD}$ with a cutoff value of $<8.73$ (sensitivity $91.1 \%$, specificity $97.8 \%$ ) (Figure 2 ). The correlation analysis between redox markers and MMSE has shown no significant relationship (GSH $r=-0.215 ; P>0.05$; GSSG $r=-0.148 ; P>0.05$ and GSH/GSSG ratio $r=0.002 ; P>0.05)$.

\section{DISCUSSION}

Oxidative stress plays a pivotal role in driving the pathological process of $\mathrm{AD}$. While the symptoms of $\mathrm{AD}$ appear later in life,

TABLE 2 | Plasma levels of amyloid, tau, and tau/amyloid ratio.

\begin{tabular}{lrcr}
\hline Subject & Amyloid, pg/ml & Tau, pg/ml & Tau/amyloid ratio \\
\hline Alzheimer's disease & $174.87 \pm 62.15$ & $451.76 \pm 240.82$ & $3.08 \pm 2.35$ \\
Control & $90.62 \pm 42.35$ & $836.93 \pm 369.31$ & $13.36 \pm 4.42$ \\
\hline
\end{tabular}

The values are articulated as mean $\pm S D$ 

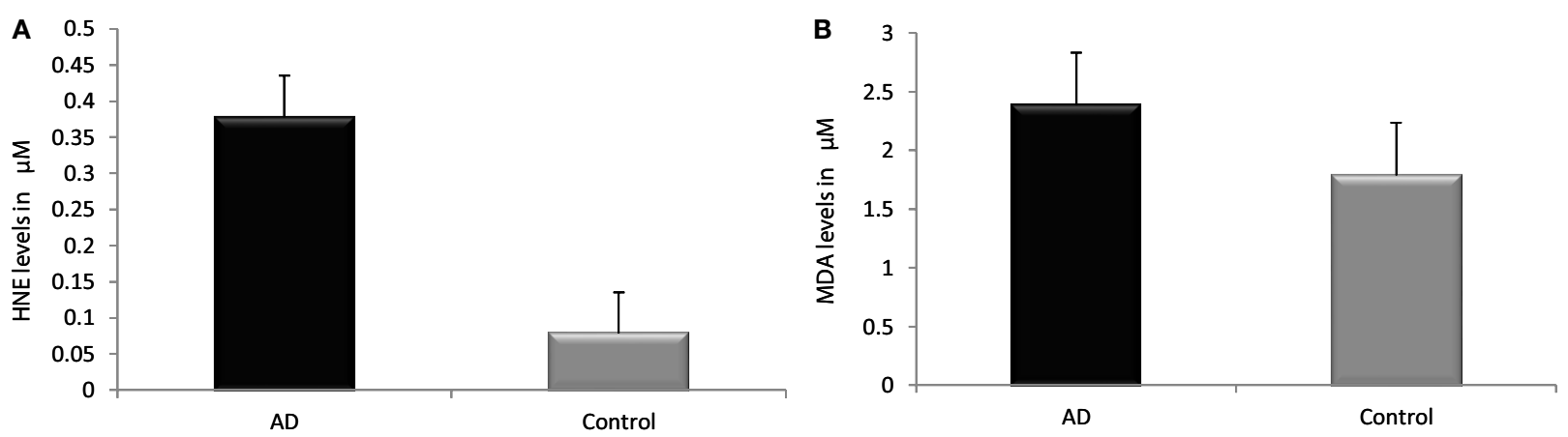

FIGURE 1 | Assessment of lipid peroxidation markers. (A) Hydroxynonenal, (B) malondialdehyde in plasma of Alzheimer's disease and control. Data represents the mean $\pm \mathrm{SD}$. ${ }^{\star} P<0.001$, significantly different from control group.
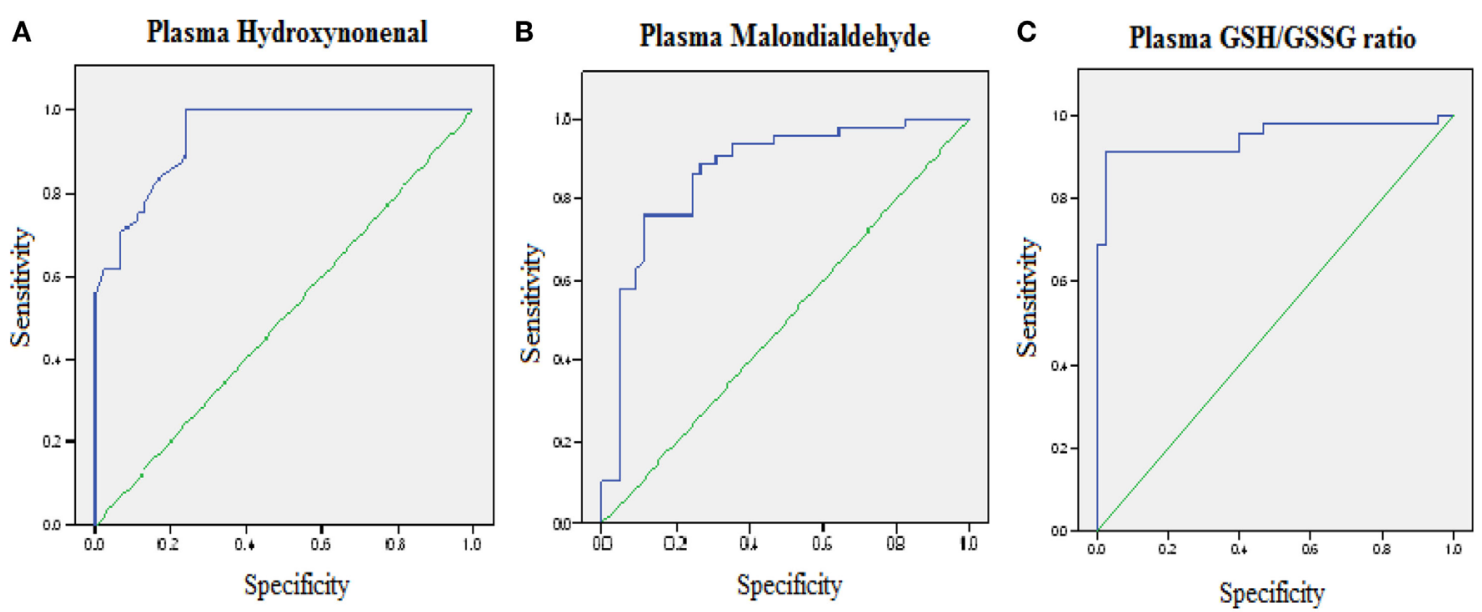

FIGURE 2 | Receiver operating characteristic curve analysis. (A) Hydroxynonenal-area under the curve (AUC): 0.93 (P < 0.01$)$, cutoff: >0.1057 $\mu$ M, sensitivity: 93.5\%, specificity: 75\%; (B) malondialdehyde-AUC: 0.87 ( $P<0.01)$, cutoff: $>1.98 \mu \mathrm{M}$, sensitivity: $80 \%$, specificity: $75 \%$; (C) GSH/GSSG ratio-AUC: 0.95 $(P<0.01)$, cutoff: <8.73, sensitivity: $91.1 \%$, specificity: $97.8 \%$. The AUC determines the quality of diagnostic test: $0.9-1$ excellent, $0.8-0.9$ good, $0.7-0.8$ fair, 0.6-0.7 poor, 0.5-0.6 fail.

the oxidative damage to the neurons starts decades, before the onset of the actual symptoms (6). Since timely diagnosis is imperative to monitor the progression of the disease and to prevent further damage, identifying reliable peripheral markers are of utmost importance. In the present study, we have evaluated the LPO indicators: HNE and MDA, redox peptides: GSH and GSSG, in order to validate their use as potential oxidative stress markers of AD by correlating their levels with known pathological markers namely A $\beta 42$ and total tau in AD blood samples.

The well-known pathological markers of Alzheimer's disease (AD) are extracellular amyloid beta peptide (A $\beta$ peptide) plaques and intracellular neurofibrillary tangles (NFTs) of hyperphosphorylated Tau (22). Elevated levels of tau, phosphorylated tau, and amyloid peptide were reported in CSF of AD patients suggesting their use as markers for clinical trials (23). However, contradictory reports exist regarding their levels in blood (24-26). Similar plasma amyloid (A $\beta 42)$ and total tau levels were also reported by Pesini et al. (27) and Sparks et al. (25) respectively. To facilitate less invasive diagnosis, peripheral blood markers are more useful than CSF markers and other expensive brain imaging techniques. Among different peripheral tissues, blood reflects the major physiological changes in various body organs and systems including brain (28).

The results of the present study indicate that plasma $\mathrm{A} \beta 42$ was increased and total tau was decreased in AD patients compared to control group with the corresponding decrease in the tau-toamyloid ratio. $A \beta$ in plasma could be influenced by the transport of $A \beta$ peptide across blood-brain barrier (BBB) (29). A $\beta$ peptide in the brain is cleared to the systemic circulation through LRP1 a low density lipoprotein receptor related protein in BBB. However transport of $\mathrm{A} \beta$ peptide into the brain is processed through receptor for advanced glycation end products RAGE (30). Hence 

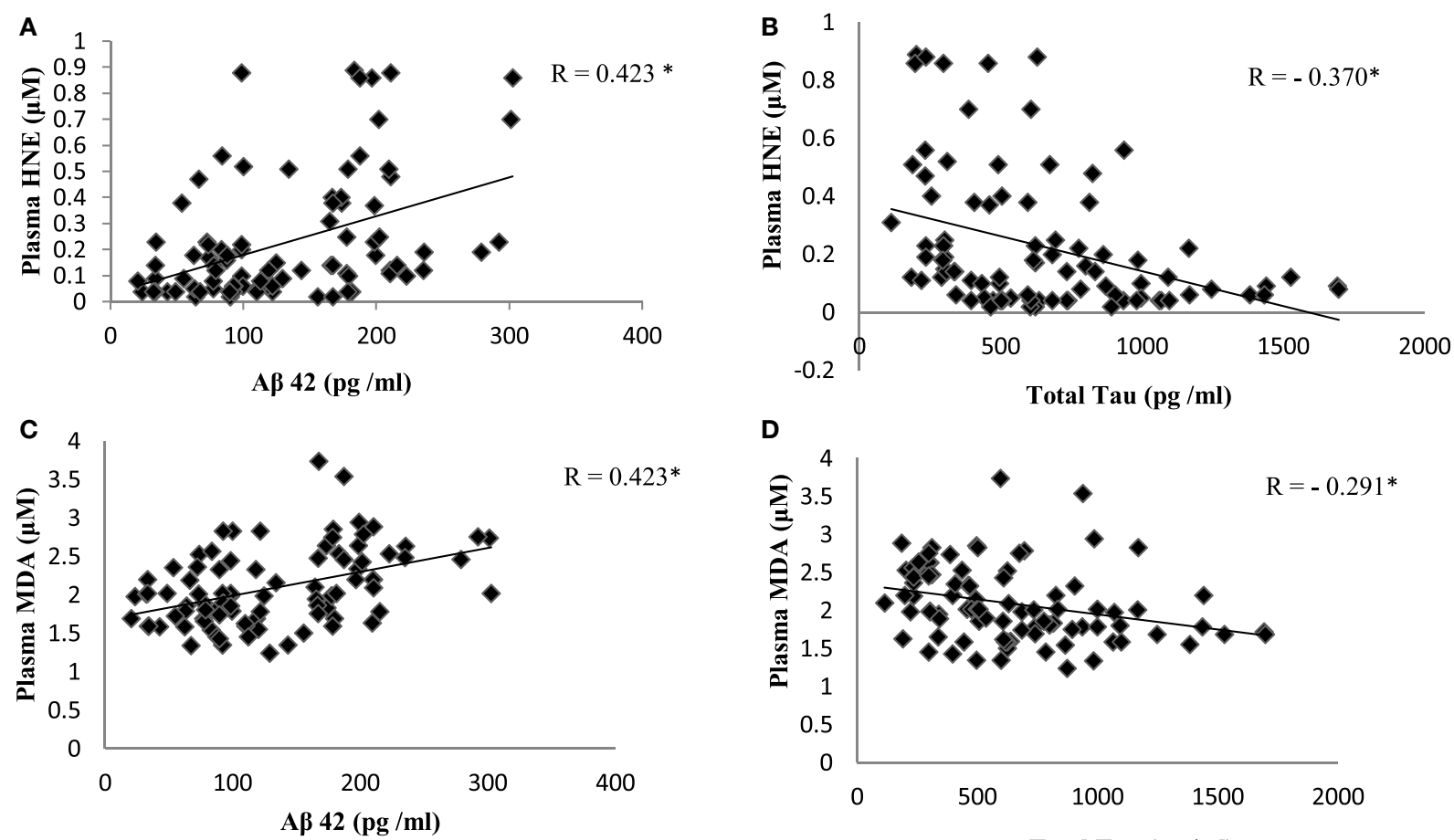

Total Tau (pg /ml)

FIGURE 3 | Correlation analysis between plasma lipid peroxidation markers and pathological markers of Alzheimer's disease: (A) hydroxynonenal (HNE) and A 342 ; (B) HNE and total tau; (C) malondialdehyde (MDA) and Aß42; (D) MDA and total tau. Correlation is significant at $P<0.01$.

the synergistic expression of LRP and RAGE could govern the transport of $\mathrm{A} \beta$ peptide across the $\mathrm{BBB}$.

Apart from brain, the tissues like skeletal muscles, platelets, kidney, pancreas, spleen and liver also contribute to the peripheral pool of $A \beta$ peptides. This limits the use of plasma $A \beta 42$ as a specific marker for AD. Neurofibrillary tangle is a hallmark of AD which occurs mainly due to abnormal phosphorylation of tau proteins. Several studies reported the elevated levels of CSF tau (31). However few reports exist regarding tau concentration in blood (32). Plog et al (33) indicated that during traumatic brain injury, tau can reach the blood via the glymphatic system. In the present study total tau level was decreased in plasma of $\mathrm{AD}$ patients compared to age- matched control group. Yet a metaanalysis study indicated that total tau in plasma alone might not be a reliable marker for diagnosis (13) and mechanistic view of tau in the peripheral pool is not well understood. However the decrease in plasma tau could be attributed due to the defective clearance of tau from the CNS to blood in AD patients. As a consequence, excess tau builds up in the CNS leading to the formation of NFTs. Sparks et al. (25) also reported a decline in cognitive function with decreased plasma total tau levels. Although contradictory reports exist regarding $A \beta$ and tau independently as plasma markers, the result of our present study indicated that the tau-to-amyloid ratio may be used as an effective marker for $\mathrm{AD}$ diagnosis (13).

Lipid peroxidation is a process, wherein the free radicals attack the lipid components resulting in the generation of lipid peroxides and hydroperoxides (34) leading to molecular cellular damage and augmenting many pathological conditions including aging. LPO results in the formation of an aldehyde such as MDA, HNE, which are of great consideration because of their high reactivity and toxicity to the biological compounds of the cell (35). Traditionally, MDA is used as a convenient biomarker for LPO but due to the non-specificity of its measurement, it is less reliable (36). However, HNE is reported as a secondary messenger of free radicals and also a major generator of oxidative stress (37). Nearly $1-8 \%$ of the HNE production in a cell is known to target the proteins (38).

Lipid peroxidation is also known to cause damage to the neuronal membrane (28) resulting in the formation of toxic secondary products, which are responsible for further cellular damage because of their ability to diffuse from the site of formation (39). Oxidative stress had been majorly implicated in $\mathrm{AD}$ pathogenesis and $\mathrm{A} \beta$ is shown to be the major source of oxidative stress in CNS (35). When neurons were treated with $\mathrm{A} \beta$, it resulted in the formation of HNE (40). Further, when NT2 neurons were exposed to HNE, enhanced production of $\mathrm{A} \beta$ was observed due to increase in activity of $\gamma$ secretase and $\beta$ secretase (41). These studies emphasis the role of oxidative stress in amyloid synthesis leading to AD. Exact role of LPO in $\mathrm{AD}$ pathology is unclear, however, increased production of amyloid as a metal chelating antioxidant could result in pathological aggregation of amyloid due to its interaction with metal ions, thereby enhancing oxidative stress leading to oxidation of lipid and other biomolecules, thereby enhancing neurotoxicity (42). 
Hydroxynonenal is known to form protein adducts, which get deposited in the various region of the brain and is also shown to diffuse across $\mathrm{BBB}$, thereby forming protein adducts in body fluids like CSF and blood (43). Elevated levels of HNE and MDA

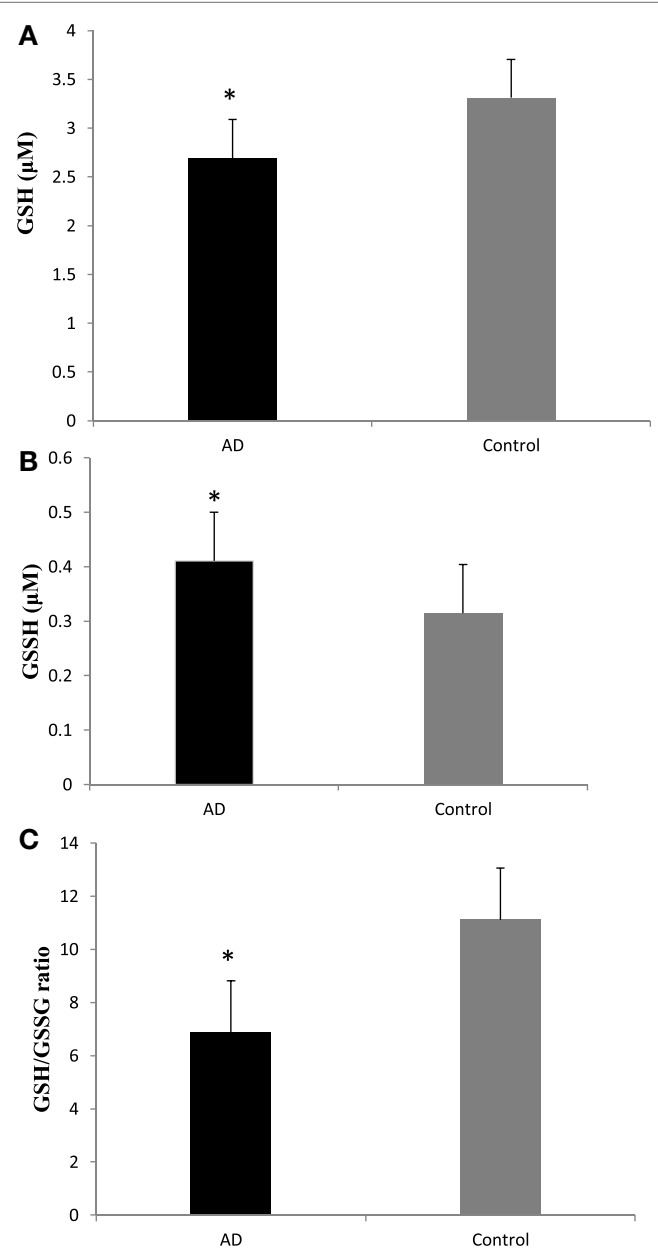

FIGURE 4 | Plasma redox status in Alzheimer's disease patients (A) GSH, (B), GSSG (C), and GSH/GSSG ratio. Data represents the mean \pm SD ${ }^{\star} P<0.001$, significantly different from control group. were reported in the brain $(10,44)$ and in erythrocytes of $\mathrm{AD}$ patients $(1,9-11)$. The present study also indicates increased levels of $\mathrm{HNE}$ and MDA in the blood of $\mathrm{AD}$ patients compared to age-matched controls. ROC analysis also revealed that HNE could be an effective marker for AD diagnosis. Studies indicated that the abnormal small vessel structure mainly affecting the endothelium occurs in $\mathrm{AD}$ leading to abnormal $\mathrm{BBB}$ function (45). This might have contributed to the elevated levels of blood $\mathrm{HNE}$ in $\mathrm{AD}$ patients by the transport of HNE from brain to blood. Positive correlation observed between plasma HNE levels and $\mathrm{AD}$ pathological markers exemplifies the same.

GSH plays a fundamental role in detoxification of ROS and also maintains intracellular redox environment of the cell effectively. GSH is also reported as a neuroprotectant by nullifying the toxic effect of ROS in neuronal cells $(46,47)$. Depletion of GSH observed in the brain of AD patients and also in AD animal models indicates the protective role of GSH in the age-related neurodegenerative disorders. A balance between GSH and GSSG levels are maintained by the enzymes glutathione peroxidase (GPx) and GR. Gpx scavenges the excess ROS produced as a result of excessive oxidative stress by oxidizing GSH to GSSG. This is reversed by GR, thereby maintaining the levels of GSH in the cells. Numerous studies have reported an increase in the activity of GPx and decreased GR activity in AD patients, thereby altering the levels of GSH $(8,9)$. The reduction in the levels of GSH with an increase in GSSG observed in blood samples of AD patients is attributed mainly due to the alteration in the activity of GPx and GR as reported earlier in our study (9). A decrease in GSH/GSSG ratio was also observed in both blood and brain of AD mice (48). The results of the present study indicates pertubance in redox status and alteration in pathological markers in plasma of $\mathrm{AD}$ patients; however, these markers are not associated with MMSE scores since plasma levels of these constituents are also influenced by other peripheral tissue sources, thereby limiting their use as specific diagnostic marker for AD.

Taking consideration of oxidative damage in $\mathrm{AD}$ pathology, many studies have focused on use of antioxidants as potential therapeutics. Most antioxidant drugs established general success in animal models, however, shown less beneficial effects in human trials; clinical trials for $\mathrm{AD}$ prevention by antioxidants supplements are still in initial stage and further studies are quite
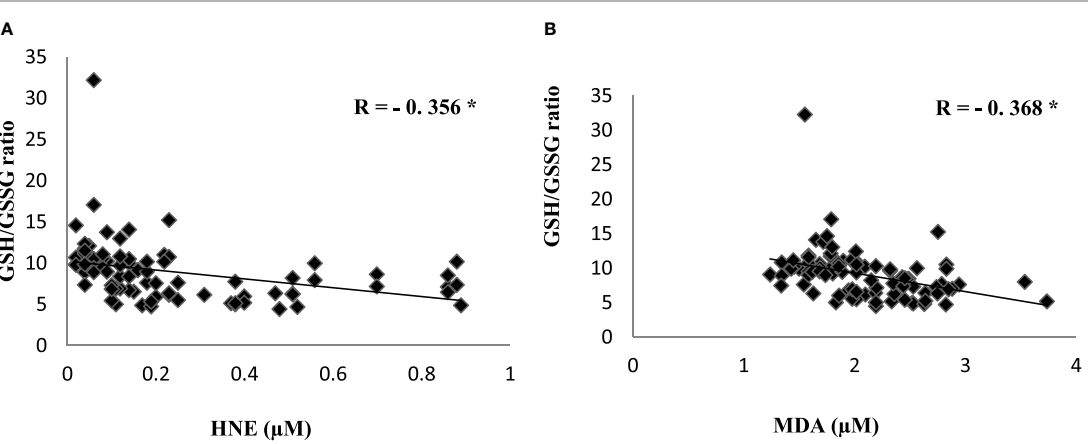

FIGURE 5 | Correlation analysis of redox markers with lipid peroxidation markers in Alzheimer's disease. (A) HNE, (B) malondialdehyde. Correlation is significant at $P<0.001$. 

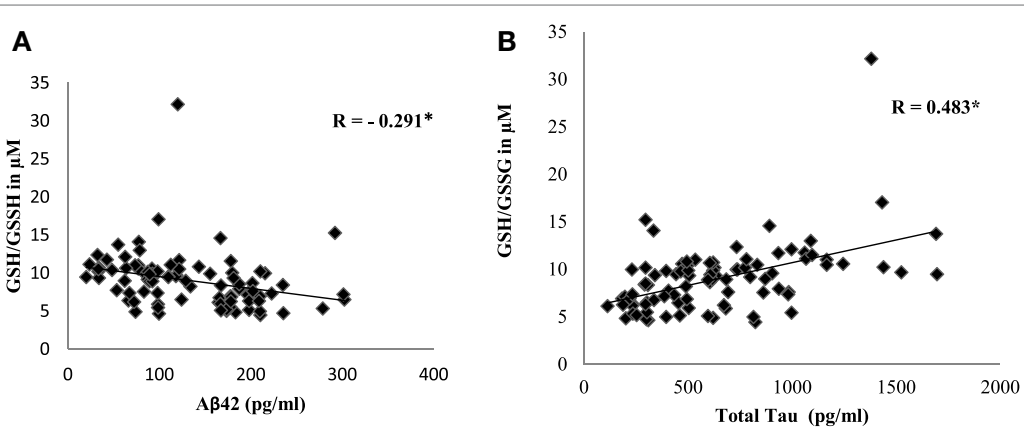

FIGURE 6 | Correlation analysis of GSH/GSSH ratio with pathological markers in Alzheimer's disease. (A) Aß42, (B) total tau. Correlation is significant at $P<0.001$.

necessary to determine if antioxidants may decrease the risk or slow the progression of the disease for AD patients (49).

Alteration in redox homeostasis leads to the modification of protein like oxidation and $S$-glutathionylation. $S$-glutathionylated actin was reported in AD brain by DalleDonne et al. (50). Other proteins such as GAPDH, hemoglobin, crystalline $\mathrm{B}, \alpha$ enolase also shown to be thiolated, causing a disturbance in glucose metabolism and oxygen supply in neurons $(12,51)$. In the present study, alteration in redox status along with enhanced LPO observed in the blood of AD patients might also contribute to thiolation of crucial blood proteins. Further studies are warranted to investigate the exact mechanism behind the protein thiolation due to elevated LPO.

\section{ETHICS STATEMENT}

This study was carried out in accordance with the recommendations from Institutional Ethical Committee, PSG Institute of Medical Sciences and Research (PSG IMS\&R), Coimbatore, and Madras Medical College and Government General Hospital (MMC), Chennai with written informed consent from all subjects. All subjects gave written informed consent

\section{REFERENCES}

1. Jomova K, Vondrakova D, Lawson M, Valko M. Metals, oxidative stress and neurodegenerative disorders. Mol Cell Biochem (2010) 345:91-104. doi:10.1007/s11010-010-0563-x

2. Kritchevsky SB, Muldoon MF. Oxidative stress and aging: still a hypothesis. J Am Geriatr Soc (1996) 44:873-5. doi:10.1111/j.1532-5415.1996.tb03751.x

3. Chatterjee SN, Agarwal S. Liposomes as membrane model for study of lipid peroxidation. Free Radic Biol Med (1988) 4:51-72. doi:10.1016/0891-5849(88)90011-1

4. Steinberg D. Low density lipoprotein oxidation and its pathobiological significance. J Biol Chem (1997) 272:20963-6. doi:10.1074/ jbc.272.34.20963

5. Sayre LM, Smith MA, Perry G. Chemistry and biochemistry of oxidative stress in neurodegenerative disease. Curr Med Chem (2001) 8:721-38. doi:10.2174/0929867013372922

6. Butterfield DA, Reed T, Sultana R. Roles of 3-nitrotyrosine- and 4-hydroxynonenal-modified brain proteins in the progression and pathogenesis of Alzheimer's disease. Free Radic Res (2011) 45:59-72. doi:10.3109/10715762. 2010.520014 in accordance with the Declaration of Helsinki. The protocol was approved by the Institutional Ethical Committee of PSG IMSR and MMC.

\section{AUTHOR CONTRIBUTIONS}

PR, SK, RC: conception and design of study; acquisition of data; analysis and/or interpretation of data; drafting the manuscript; revising the manuscript critically for important intellectual content; approval of the version of the manuscript to be published.

\section{ACKNOWLEDGMENTS}

The authors thank Dr. M. B. Pranesh, PSG IMS\&R, Coimbatore, and ARDSI, Thrissur, for referring patients and Department of Biotechnology (DBT), New Delhi for financial support.

\section{FUNDING}

The study was supported by Department of Biotechnology (DBT), Ministry of Science and Technology, New Delhi. BT/PR3869/ MED/30/667/2011.

7. López N, Tormo C, De Blas I, Llinares I, Alom J. Oxidative stress in Alzheimer's disease and mild cognitive impairment with high sensitivity and specificity. J Alzheimers Dis (2013) 33:823-9. doi:10.3233/JAD-2012-121528

8. Torres LL, Quaglio NB, de Souza GT, Garcia RT, Dati LM, Moreira WL, et al. Peripheral oxidative stress biomarkers in mild cognitive impairment and Alzheimer's disease. J Alzheimers Dis (2011) 26:59-68. doi:10.3233/ JAD-2011-110284

9. Krishnan S, Rani P. Evaluation of selenium, redox status and their association with plasma amyloid/tau in Alzheimer's disease. Biol Trace Elem Res (2014) 158(2):158-65. doi:10.1007/s12011-014-9930-x

10. Markesbery WR, Lovell MA. Four-hydroxynonenal, a product of lipid peroxidation, is increased in the brain in Alzheimer's disease. Neurobiol Aging (1998) 19:33-6. doi:10.1016/S0197-4580(98)00009-8

11. Mandal PK, Tripathi M, Sugunan S. Brain oxidative stress: detection and mapping of anti-oxidant marker 'Glutathione' in different brain regions of healthy male/female, MCI and Alzheimer patients using non-invasive magnetic resonance spectroscopy. Biochem Biophys Res Commun (2012) 417(1):43-8. doi:10.1016/j.bbrc.2011.11.047

12. Newman SF, Sultana R, Perluigi M, Coccia R, Cai J, Pierce WM, et al. An increase in $S$-glutathionylated proteins in the Alzheimer's disease inferior 
parietal lobule, a proteomics approach. J Neurosci Res (2007) 85(7):1506-14. doi:10.1002/jnr.21275

13. Shanthi KB, Krishnan S, Rani P. A systematic review and meta-analysis of plasma amyloid 1-42 and tau as biomarkers for Alzheimer's disease. SAGE Open Med (2015) 3. doi:10.1177/2050312115598250

14. Folstein MF, Folstein SE, Mc Hugh PR. Mini-mental state: a practical method for grading the cognitive state of patients for the clinician. J Psychiatr Res (1975) 12:189-98. doi:10.1016/0022-3956(75)90026-6

15. McKhann G, Drachman D, Folstein M, Katzman R, Price D, Stadlan EM. Clinical diagnosis of Alzheimer's disease: report of the NINCDS-ADRDA Work Group under the auspices of Department of Health and Human Services Task Force on Alzheimer's Disease. Neurology (1984) 34:939-44. doi:10.1212/WNL.34.7.939

16. Buege JA, Aust SD. Microsomal lipid peroxidation. Methods Enzymol (1978) 52:302-10. doi:10.1016/S0076-6879(78)52032-6

17. Benedetti A, Comporti M, Esterbauer H. Identification of 4-hydroxynonenal as a cytotoxic product originating from the peroxidation of liver microsomal lipids. Biochim Biophys Acta (1980) 620(2):281-96. doi:10.1016/0005-2760(80)90209-x

18. Ellman GL. Tissue sulfhydryl groups. Arch Biochem Biophys (1959) 82(1):70-7. doi:10.1016/0003-9861(59)90090-6

19. Owens C, Belcher R. A colorimetric micro-method for the determination of glutathione. Biochem J (1965) 94(3):705-11. doi:10.1042/bj0940705

20. Skoumalová A, Hort J. Blood markers of oxidative stress in Alzheimer's disease. J Cell Mol Med (2012) 16(10):2291-300. doi:10.1111/j.1582-4934.2012.01585.x

21. Andreasen N, Minthon L, Davidsson P, Vanmechelen E, Vanderstichele H, Winblad B. Evaluation of CSF-tau and CSF-A $\beta 42$ as diagnostic markers for Alzheimer disease in clinical practice. Arch Neurol (2001) 58(3):373-9. doi:10.1001/archneur.58.3.373

22. Blennow K, Hampel H, Weiner M, Zetterberg H. Cerebrospinal fluid and plasma biomarkers in Alzheimer disease. Nat Rev Neurol (2010) 6:131-44. doi:10.1038/nrneurol.2010.4

23. Cummings JL. Biomarkers in Alzheimer's disease drug development. Alzheimers Dement (2011) 7(3):e13-44. doi:10.1016/j.jalz.2010.06.004

24. Mayeux R, Honig LS, Tang M, Manly J, Stern Y, Schupf N, et al. Plasma A 40 and A 42 and Alzheimer's disease: relation to age, mortality, and risk. Neurology (2003) 61(9):1185-90. doi:10.1212/01.wnl.0000091890.32140.8f

25. Sparks DL, Kryscio RJ, Sabbagh MN, Ziolkowski C, Lin Y, Sparks LM, et al. Tau is reduced in AD plasma and validation of employed ELISA methods. Am J Neurodegener Dis (2012) 1(1):99-106.

26. Sunderland T, Linker G, Mirza N, Putnam KT, Friedman DL, Kimmel LH, et al. Decreased $\beta$-amyloid 1-42 and increased Tau levels in cerebrospinal fluid of patients with Alzheimer disease. JAMA (2003) 289(16):2094-103. doi:10.1001/jama.289.16.2094

27. Pesini P, Pérez-Grijalba V, Monleón I, Boada M, Tárraga L, Martínez-Lage P, et al. Reliable measurements of the $\beta$-amyloid pool in blood could help in the early diagnosis of AD. Int J Alzheimers Dis (2012) 2012(604141):10. doi:10.1155/2012/604141

28. Hye A, Lynham S, Thambisetty M, Causevic M, Campbell J, Byers HL, et al. Proteome-based plasma biomarkers for Alzheimer's disease. Brain (2006) 129(11):3042-50. doi:10.1093/brain/awl279

29. Deane R, Bell R, Sagare A, Zlokovic B. Clearance of amyloid- $\beta$ peptide across the blood-brain barrier: implication for therapies in Alzheimers disease. Curr Drug Targets CNS Neurol Disord (2009) 8(1):16-30. doi:10.2174/187152709787601867

30. Deane R, Wu Z, Sagare A, Davis J, Du Yan S, Hamm K, et al. LRP/amyloid $\beta$-peptide interaction mediates differential brain efflux of $A \beta$ isoforms. Neuron (2004) 43(3):333-44. doi:10.1016/j.neuron.2004.07.017

31. Blennow K, Vanmechelen E, Hampel H. CSF total tau, A $\beta 42$ and phosphorylated tau protein as biomarkers for Alzheimer's disease. Mol Neurobiol (2001) 24(1-3):087-98. doi:10.1385/mn:24:1-3:087

32. Zetterberg H, Wilson D, Andreasson U, Minthon L, Blennow K, Randall J, et al. Plasma tau levels in Alzheimer's disease. Alzheimers Res Ther (2013) 5(2):9. doi:10.1186/alzrt163

33. Plog BA, Dashnaw ML, Hitomi E, Peng W, Liao Y, Lou N. Biomarkers of traumatic injury are transported from brain to blood via the glymphatic system. J Neurosci (2015) 35(2):518-26. doi:10.1523/jneurosci.3742-14.2015
34. Yin $\mathrm{H}, \mathrm{Xu} \mathrm{L}$, Porter NA. Free radical lipid peroxidation: mechanisms and analysis. Chem Rev (2011) 111(10):5944-72. doi:10.1021/cr200084z

35. Esterbauer H, Cheeseman KH. Determination of aldehydic lipid peroxidation products: malonaldehyde and 4-hydroxynonenal. Methods Enzymol (1990) 186:407-21. doi:10.1016/0076-6879(90)86134-H

36. McGrath LT, McGleenon BM, Brennan S, McColl D, McILroy S, Passmore AP. Increased oxidative stress in Alzheimer's disease as assessed with 4-hydroxynonenal but not malondialdehyde. QJM (2001) 94(9):485-90. doi:10.1093/qjmed/94.9.485

37. Zarkovic N. 4-Hydroxynonenal as a bioactive marker of pathophysiological processes. Mol Aspects Med (2003) 24(4-5):281-91. doi:10.1016/ s0098-2997(03)00023-2

38. Perluigi M, Coccia R, Butterfield DA. 4-Hydroxy-2-nonenal, a reactive product of lipid peroxidation, and neurodegenerative diseases: a toxic combination illuminated by redox proteomics studies. Antioxid Redox Signal (2012) 17(11):1590-609. doi:10.1089/ars.2011.4406

39. Yehuda S, Rabinovitz S, Carasso RL, Mostofsky DI. The role of polyunsaturated fatty acids in restoring the aging neuronal membrane. Neurobiol Aging (2002) 23(5):843-53. doi:10.1016/s0197-4580(02)00074-x

40. Mark RJ, Lovell MA, Markesbery WR, Uchida K, Mattson MP. A role for 4-hydroxynonenal, an aldehydic product of lipid peroxidation, in disruption of ion homeostasis and neuronal death induced by amyloid $\beta$-peptide. J Neurochem (2002) 68(1):255-64. doi:10.1046/j.1471-4159.1997.68010255.x

41. Tamagno E, Bardini P, Obbili A, Vitali A, Borghi R, Zaccheo D, et al. Oxidative stress increases expression and activity of BACE in NT2 neurons. Neurobiol Dis (2002) 10(3):279-88. doi:10.1006/nbdi.2002.0515

42. Arlta S, Beisiegela U, Kontushb A. Lipid peroxidation in neurodegeneration: new insights into Alzheimer's disease. Curr Opin Lipidol (2002) 13(3):289-94. doi:10.1097/00041433-200206000-00009

43. Zarkovic K. 4-Hydroxynonenal and neurodegenerative diseases. Mol Aspects Med (2003) 24(4-5):293-303. doi:10.1016/s0098-2997(03)00024-4

44. Balazs L, Leon M. Evidence of an oxidative challenge in the Alzheimer's brain. Neurochem Res (1994) 19(9):1131-7. doi:10.1007/bf00965146

45. Kelleher RJ, Soiza RL. Evidence of endothelial dysfunction in the development of Alzheimer's disease: is Alzheimer's a vascular disorder? Am J Cardiovasc Dis (2013) 3(4):197-226.

46. Dringen R. Metabolism and functions of glutathione in brain. Prog Neurobiol (2000) 62:649-71. doi:10.1016/s0301-0082(99)00060-x

47. Schulz JB, Lindenau J, Seyfried J, Dichgans J. Glutathione, oxidative stress and neurodegeneration. Eur JBiochem (2000) 267:4904-11. doi:10.1046/j.1432-1327.2000.01595.x

48. Zhang C, Rodriguez C, Spaulding J, Aw TY, Feng J. Age-dependent and tissue-related glutathione redox status in a mouse model of Alzheimer's disease. J Alzheimers Dis (2014) 28(3):655-66. doi:10.3233/JAD-2011-111244

49. Feng Y, Wang X. Antioxidant therapies for Alzheimer's disease. Oxid Med Cell Longev (2012) 2012:1-17. doi:10.1155/2012/472932

50. Dalle-Donne I, Rossi R, Giustarini D, Colombo R, Milzani A. Actin $S$-glutathionylation: evidence against a thiol-disulphide exchange mechanism. Free Radic Biol Med (2003) 35(10):1185-93. doi:10.1016/s0891-5849(03) 00504-5

51. Dinoto L, Deture MA, Purich DL. Structural insights into Alzheimer filament assembly pathways based on site-directed mutagenesis and $S$-glutathionylation of three-repeat neuronal Tau protein. Microsc Res Tech (2005) 67(3-4):156-63. doi:10.1002/jemt.20195

Conflict of Interest Statement: The authors declare that the research was conducted in the absence of any commercial or financial relationships that could be construed as a potential conflict of interest.

Copyright $\odot 2017$ Rani, Krishnan and Rani Cathrine. This is an open-access article distributed under the terms of the Creative Commons Attribution License (CC BY). The use, distribution or reproduction in other forums is permitted, provided the original author(s) or licensor are credited and that the original publication in this journal is cited, in accordance with accepted academic practice. No use, distribution or reproduction is permitted which does not comply with these terms. 